\title{
Erlotinib Activates Different Cell Death Pathways in EGFR-mutant Lung Cancer Cells Grown in 3D Versus 2D Culture Systems
}

\author{
HYUN-KYUNG LEE ${ }^{1 *}$, MIN HYE NOH ${ }^{2 *}$, SEUNG-WOO HONG ${ }^{2 *}$, SEUNG-MI KIM ${ }^{2}$, \\ SUNG HYUN KIM ${ }^{1}$, YEONG SEOK KIM ${ }^{2}$, V. COURTNEY BROADDUS ${ }^{3}$ and DAE YOUNG HUR ${ }^{2}$ \\ ${ }^{1}$ Division of Pulmonary, Allergy, and Critical Care Medicine, \\ Department of Internal Medicine, Inje University Busan Paik Hospital, Busan, Republic of Korea; \\ ${ }^{2}$ Department of Anatomy and Tumor Immunology, Inje University College of Medicine, Busan, Republic of Korea; \\ ${ }^{3}$ Division of Pulmonary, Critical Care, Allergy and Sleep Medicine, Department of Medicine, \\ Zuckerberg San Francisco General Hospital and Trauma Center, \\ University of California San Francisco, San Francisco, CA, U.S.A.
}

\begin{abstract}
Background/Aim: Non-small cell lung cancer patients with epidermal growth factor receptor (EGFR) mutation have been shown to have a good response to erlotinib, a receptor tyrosine kinase inhibitor of EGFR. In this study, we found that the cell death pathways activated by erlotinib in $2 D$ and $3 D$ culture systems are different. Materials and Methods: The cell death pathways induced by erlotinib were evaluated by flow cytometry and immunoblotting in both $2 D$ and $3 D$ culture systems of EGFR mutant lung cancer cells. Results: Treatment with erlotinib induced caspase 8 activation and up-regulation of TNFrelated apoptosis-inducing ligand (TRAIL) expression only in $3 D$ cultures. Knockdown of TRAIL attenuated both erlotinib-induced activation of caspase- 8 and apoptosis in $3 D$ cultures. Erlotinib also increased LC3, an autophagy marker, expression and c-Jun $N$ terminal kinase (JNK) activation. Both 3-MA as an autophagy inhibitor and
\end{abstract}

This article is freely accessible online.

*These Authors contributed equally to this work.

Correspondence to: Dae Young Hur, MD, Ph.D., Department of Anatomy and Tumor Immunology, Inje University College of Medicine, 75 Bokji-ro, Busanjin-gu, Busan 47392, Republic of Korea. Tel: +82 518906796, Fax: +82 518966634, e-mail: dyhur@inje.ac.kr and V. Courtney Broaddus, MD, Ph.D., Division of Pulmonary, Critical Care, Allergy and Sleep Medicine, Department of Medicine, Zuckerberg San Francisco General Hospital and Trauma Center, University of California San Francisco, San Francisco, CA 94110, U.S.A. Tel: +1 4152063513, Fax: +1 4156951551, e-mail: Courtney.Broaddus@ucsf.edu

Key Words: Erlotinib, 3D spheroid, TRAIL, autophagy, lung cancer.
SP600125 as a JNK inhibitor, significantly inhibited erlotinib-induced cell death. Conclusion: Erlotinib induces apoptotic cell death in $3 D$ cultures through an autophagyTRAIL-JNK pathway.

Lung cancer was the most common cause of cancer-related death in males and the second most common cause of death in females world-wide in 2018 (1). Receptor tyrosine kinases such as epidermal growth factor receptor (EGFR) and anaplastic lymphoma kinase (ALK) are dysregulated and oncogenic in some non-small cell lung cancer (NSCLC) cases (2, 3). Among these tyrosine kinases, EGFR has wellcharacterized roles in cell proliferation, survival, invasion, and metastasis, through gene amplification or gain-offunction mutations, such as the T790 mutation in the EGFR kinase domain $(4,5)$. This dysregulation of EGFR results in oncogenic transformation, and patients with mutations in this gene have a poorer survival rate than patients without mutations (6).

Erlotinib, which inhibits tyrosine kinase activity by binding to the ATP-binding pocket of EGFR, has been approved and is now widely used to treat NSCLC patients with EGFR mutation (7). Molecular mechanisms underlying cell death by erlotinib have been described for standard 2D monolayer NSCLC cultures. In this setting, EGFR inhibition by erlotinib induces Bcl-2 interacting mediator of cell death (BIM)- or reactive-oxygen species (ROS)-dependent apoptotic cell death $(8,9)$. However, in 3D NSCLC cell cultures, the mechanism by which erlotinib induces cell death has yet to be explored, even though this information could potentially inform novel anti-cancer therapies for NSCLC patients.

Three-dimensional spheroid cell culture systems, which have been shown to mimic the in vivo setting and resemble the tumor microenvironment better than 2D culture systems, 
have been utilized as anti-cancer drug testing platforms and a screening system for several cancers $(10,11)$. These studies found that $2 \mathrm{D}$ lung and mesothelioma cancer cells were generally more sensitive to typical anti-cancer chemo-agents or targeted agents than the corresponding 3D cells (12-14). However, whether the mechanisms of cell death by anticancer drugs differ between 2D and 3D cell culture systems is not well understood.

Autophagy, a cell degradation pathway that has either a tumor suppressor or oncogenic role in various cancer cells $(15,16)$, has been reported to play an important role in EGFR inhibitor-induced cell death $(17,18)$. Chen et al. suggested that autophagy initiated by gefitinib, another EGFR inhibitor, up-regulated TRAIL and induced apoptotic cell death (17). However, another group found that autophagy suppressed erlotinib-induced cell death in NSCLC. Autophagy, which has been shown to be enhanced in the 3D setting (17), could play a role in EGFR inhibition and cell death in $3 \mathrm{D}$, but not $2 \mathrm{D}$ systems.

In this study, we investigated the mechanisms of cell death by erlotinib in NSCLC cells with EGFR mutations grown under $2 \mathrm{D}$ and $3 \mathrm{D}$ culture conditions. We found that erlotinib activates an autophagy-TRAIL-JNK pathway in the 3D culture system, but not in the $2 \mathrm{D}$ culture system. In other words, the cell death pathway activated by erlotinib differs between $2 \mathrm{D}$ and $3 \mathrm{D}$ culture systems.

\section{Materials and Methods}

Cell culture and reagents. NSCLC cell lines, HCC827 and HCC4006, were purchased from ATCC (American Type Culture Collection, Manassas, VA, USA). Cells were maintained in RPMI1640 medium (Mediatech Inc., Corning Subsidiary, Manassas, VA, USA) containing heat-inactivated $10 \%$ fetal bovine serum (FBS; Tissue Culture Biologicals, Tulare, CA, USA) and $100 \mu \mathrm{g} / \mathrm{ml}$ penicillin/streptomycin (Sigma, St. Louis, MO, USA) in $5 \% \mathrm{CO}_{2}$ at $37^{\circ} \mathrm{C}$. Erlotinib was purchased from Selleck (Selleck Chemicals, Houston, TX, USA). Z-VAD-fmk (z-VAD), a pan-caspase inhibitor; z-IETD-fmk (z-IETD), a caspase-8 specific inhibitor; and SP600125, a JNK inhibitor, were purchased from Sigma.

$3 D$ spheroid cell culture. Cells were cultured in poly-HEMA-coated wells as reported previously (19). Briefly, HCC827 and HCC4006 cells were plated at a density of $1-2 \times 10^{3}$ cells per well in polyHEMA (2-hydroxyethyl methacrylate, Sigma)-coated 96-well plates. Cells were incubated for $72 \mathrm{~h}$ until the formation of spheroids.

Lentiviral shRNA infection. To knock-down TRAIL expression, we used the pLKO1-based lentiviral system (Sigma). Briefly, HEK293T cells were transfected with lentiviral packaging mix (Sigma) and pLKO-1 scrambled or TRAIL shRNA (sc shRNA: 5'-AAT TCT CCG AAC GTG TCA CGT-3'; TRAIL shRNA: 5'-AAC GAG CUG AAG CAG AUG CAG-3') using Lipofectamine 2000 (Invitrogen, Carlsbad, CA, USA), according to the manufacturer's instructions. HCC 827 and HCC4006 cells were grown as 2D monolayers for 24 $\mathrm{h}$, infected with 20 MOI (multiplicity of infection) pLKO-1 scrambled or TRAIL shRNA lentivirus for $24 \mathrm{~h}$, and then re-seeded in poly-HEMA-coated $60 \mathrm{~mm}$ plates. After spheroid formation (72 h), cells were treated with $500 \mathrm{nM}$ erlotinib for another $24 \mathrm{~h}$. Validation of shRNAs was performed by Genolution (Genolution Pharmaceutical Inc., Seoul, Republic of Korea).

Cell death and Annexin- $V$ staining. For the 2D culture studies, cells were seeded at a density of $3 \times 10^{5}$ in $60 \mathrm{~mm}$ dishes for $24 \mathrm{~h}$ and treated with erlotinib at the indicated doses for $24 \mathrm{~h}$. For 3D culture studies, cells were cultivated in poly-HEMA-coated 96-wells for 72 $\mathrm{h}$ until they formed spheroids and then, incubated with erlotinib for another $24 \mathrm{~h}$. Cells were washed with PBS, trypsinized, and stained with trypan blue or annexin-V/PI double staining solution (BD Biosciences, San Jose, CA, USA), followed by fluorescenceactivated cell sorting (BD Biosciences).

Immunoblot analysis. Cells were lysed with RIPA lysis buffer (Tech \& Innovation, Kangwon, Republic of Korea) containing a protease and phosphatase inhibitor cocktail (Sigma). After cell lysis, supernatants were collected after centrifugation at 13,000 rpm for 20 min. Protein concentrations were determined using the Bradford assay (Bio-Rad, USA). Equal total amounts of cellular proteins $(20 \mu \mathrm{g}$ per lane) were separated using $8-15 \%$ sodium dodecyl sulfatepolyacrylamide gel electrophoresis (SDS-PAGE) and were transferred to an Immobilon-PVDF membrane (Millipore Corporation, Billerica, MA, USA). Membranes were blocked with 5\% skim-milk in TBS-T (Tech \& Innovation) and probed with antibodies against cleaved caspase-8, cleaved caspase-9, cleaved caspase-3, TRAIL, Fas, phospho-JNK, JNK, LC3B (Cell Signaling Technology, Beverly, MA, USA), or $\beta$-actin (Santa Cruz Biotechnology, Santa Cruz, CA, USA). Primary antibodies were detected using horseradish peroxidaseconjugated anti-mouse or anti-rabbit secondary antibodies (Cell Signaling) and an enhanced chemiluminescence detection system (Amersham, Buckinghamshire, UK).

Statistical analysis. All data were shown as the mean \pm SD. Two ANOVA analysis using SPSS 26 (SPSS Inc., Chicago, IL, USA) were performed to evaluate $p$-values, and $p$-values less than 0.05 were considered significant.

\section{Results}

Erlotinib induces caspase-8-dependent apoptotic cell death in $3 D$ culture but not in $2 D$ culture. First, we investigated the effect of erlotinib on cell viability in 2D and 3D cultures, using HCC827 and HCC4006 cells, which have been reported to form 3D spheroids (20). It was reported that HCC827 and HCC4006 cells, which have EGFR gene amplification and deletion of exon 19, are sensitive to erlotinib (21). As has been reported previously (20), we found that phosphorylation of EGFR was dramatically up-regulated in 3D cultures compared to 2D cultures in both HCC 827 and HCC4006 cells (Figure 1A and B). Moreover, as reported previously, the rate of cell death in NSCLC 2D culture cells increased significantly after exposure to erlotinib in a dose-dependent manner. Surprisingly, an increased rate of cell death was found in response to erlotinib in cells grown in 3D culture compared to 2D (Figure 1C and D). 
A

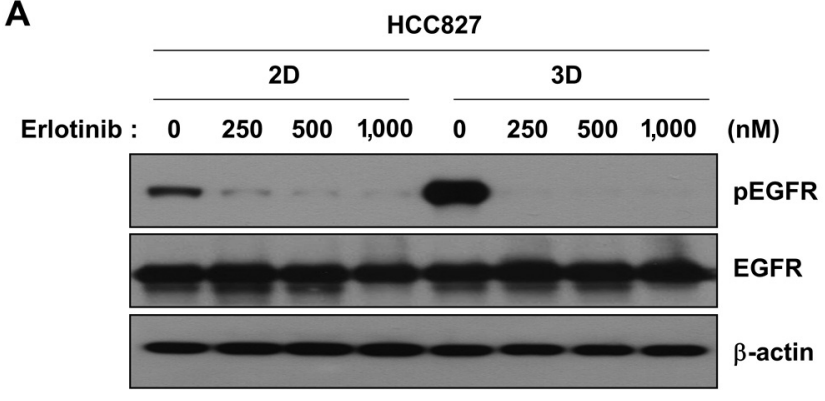

C

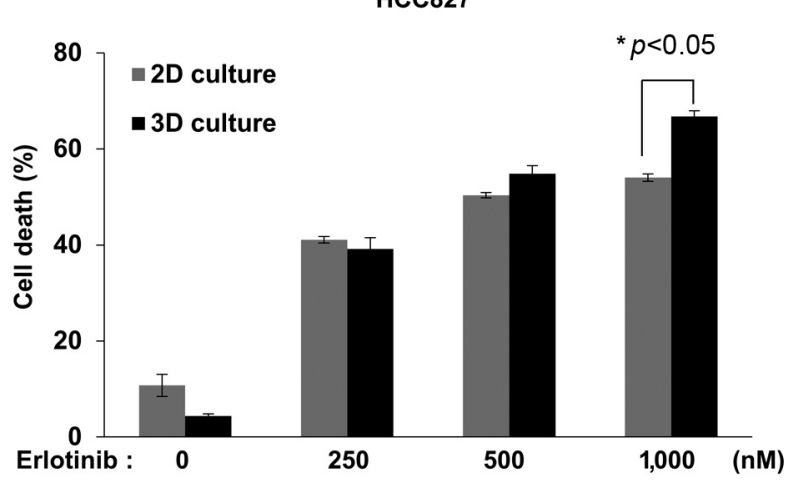

E

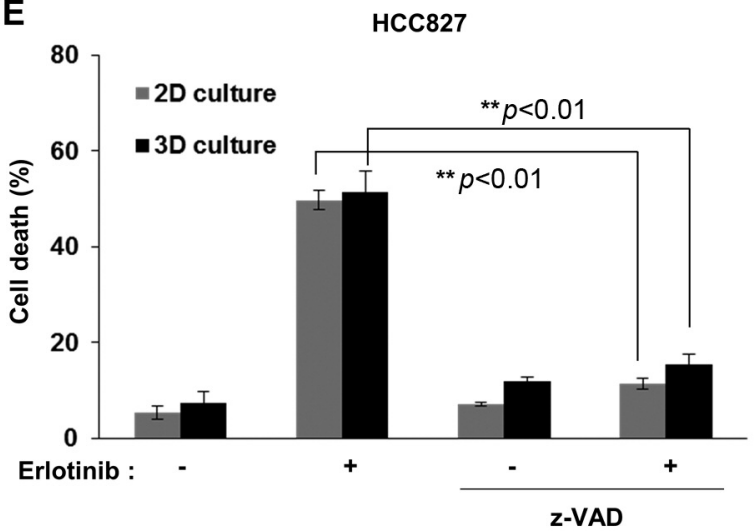

B

\begin{tabular}{ccc} 
HCC4006 \\
\hline 2D
\end{tabular}

\begin{tabular}{llllllllllll}
\cline { 2 - 4 } & Erlotinib : & 0 & 250 & 500 & 1,000 & 0 & 250 & 500 & 1,000 & (nM)
\end{tabular}

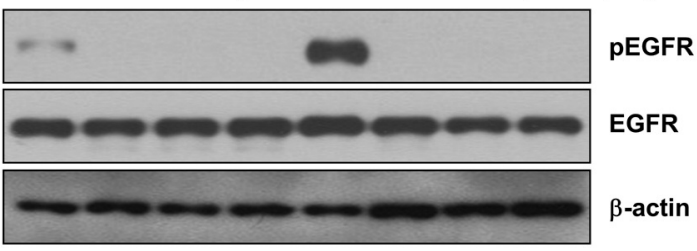

D

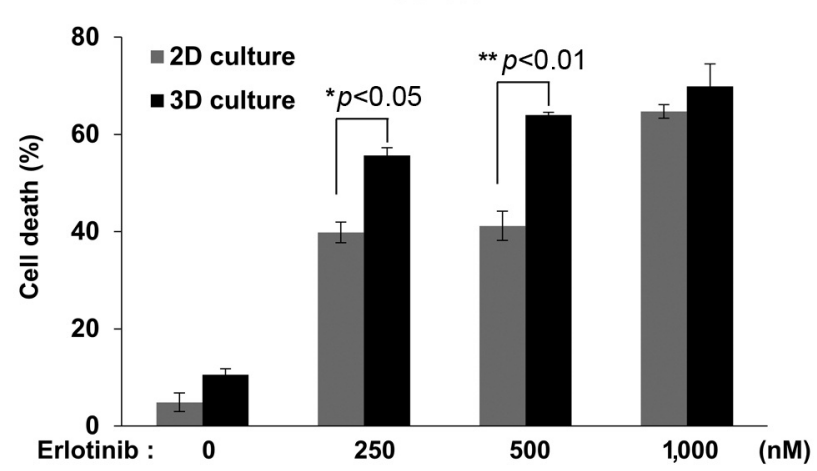

$\mathbf{F}$

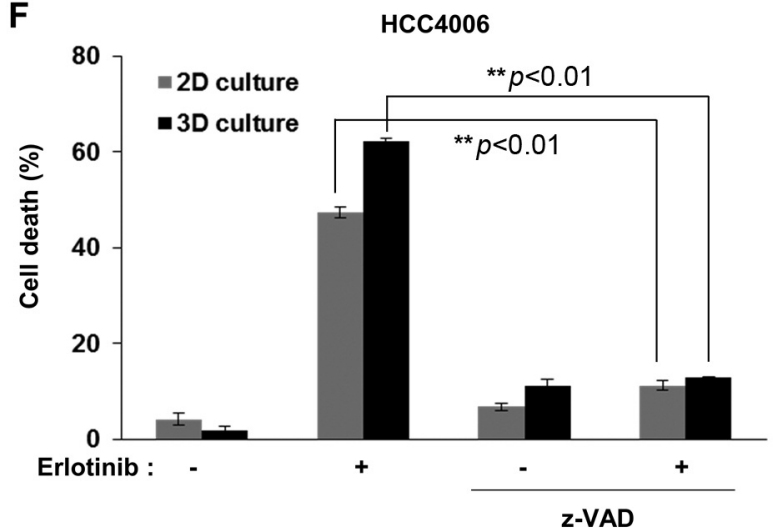

Figure 1. Erlotinib induces cell death in both $2 D$ and $3 D$ NSCLC cell culture systems. Erlotinib inhibits the phosphorylation of EGFR in both $2 D$ and $3 D$ cultures. HCC 827 and HCC4006 cells grown in $2 D$ and $3 D$ cultures were treated with erlotinib at the indicated doses. Cell lysates were prepared for immunoblot analysis using anti-p-EGFR or anti-EGFR antibodies. $\beta$-actin was used as a loading control (A and B). HCC827 and HCC4006 cells were treated with erlotinib at the indicated doses for $24 \mathrm{~h}$. Cell death was determined by the trypan blue exclusion method. Error bars represent the mean $\pm S . D$. of three separate experiments performed in triplicate $(C$ and $D)$. $3 D$ spheroid cells were pretreated with $50 \mu M z$ $V A D$ before exposure to $500 \mathrm{nM}$ erlotinib; after $24 \mathrm{~h}$, cell death was evaluated by the trypan blue method ( $E$ and $F$ ). Error bars represent mean $\pm S . D$. of three separate experiments. * and $* *$ indicate $p<0.05$ and $p<0.01$, respectively.

Next, we evaluated whether cell death by erlotinib is caspase-dependent in 3D cultures, as has been described for NSCLC cells grown in 2D culture (21). We found that cell death by erlotinib was completely abrogated by pretreatment of cells grown in 2D and 3D culture with a pan-caspase inhibitor (Figure 1E and F). These results indicate that erlotinib treatment induces caspase-dependent apoptotic cell death in both $2 \mathrm{D}$ and $3 \mathrm{D}$ cultures.

Next, we examined the effect of erlotinib on the caspase cascade in 2D and 3D cultures. Interestingly, caspase- 8 was 
A

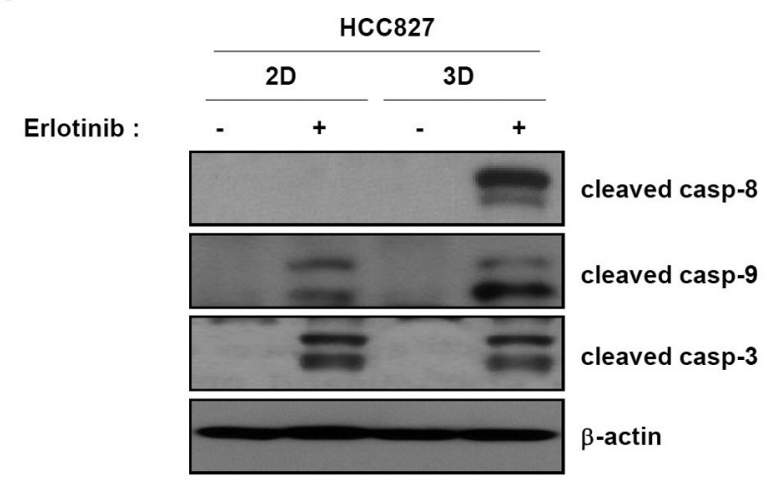

C

$\mathrm{HCC} 827$

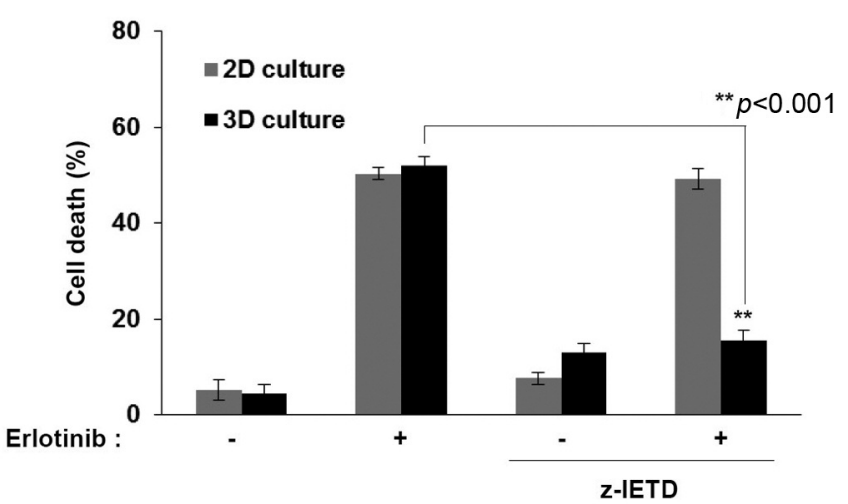

B

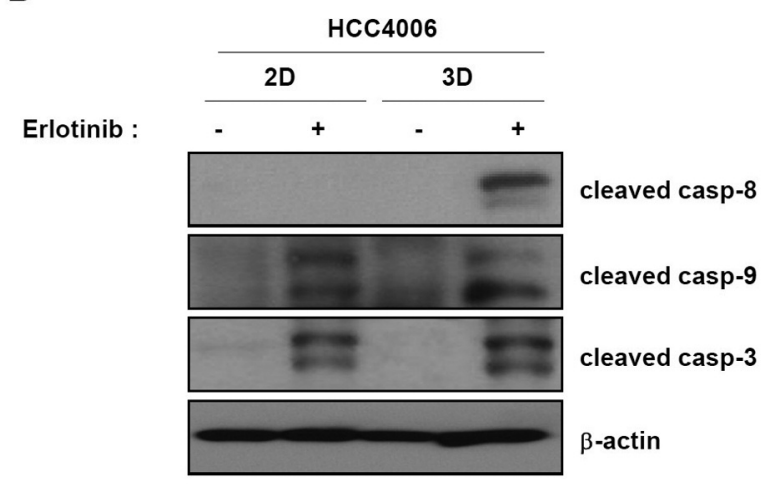

D

HCC4006

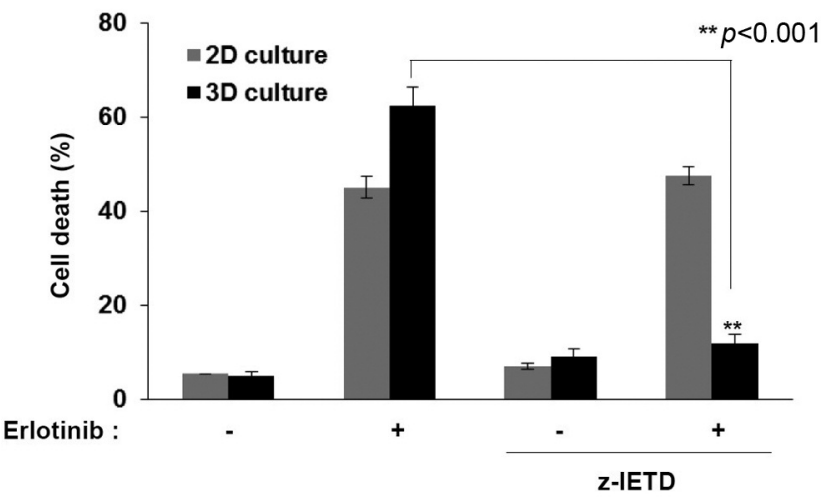

Figure 2. Erlotinib activates caspase-8 in 3D, not in the 2D, cell culture systems. (A and B) HCC827 and HCC4006 cells grown under $2 D$ and $3 D$ culture conditions were treated with $500 \mathrm{nM}$ erlotinib for $24 \mathrm{~h}$. Cell lysates were prepared for immunoblot analysis. $\beta$-actin was used as a loading control. (C and D) Cells were treated with $500 \mathrm{nM}$ erlotinib after pre-treatment with $20 \mu \mathrm{M}$-IETD, and cell death was measured by the trypan blue method. Error bars indicate mean \pm S.D. of three separate experiments. $* * p<0.01$.

activated after exposure to erlotinib in $3 \mathrm{D}$ - but not in $2 \mathrm{D}$ cultured cells (Figure 2A and B). To confirm the activation of caspase- 8 in 3D cell cultures, we tested whether treatment with a caspase- 8 inhibitor, z-IETD, decreased erlotinibinduced cell death in 3D cultures but not in 2D cultures, and found that that this was the case (Figure 2C and D). These results suggest that erlotinib induces caspase-cascade apoptotic cell death differently in 2D and 3D cell cultures.

Erlotinib up-regulates the expression of TRAIL in $3 D$ cell cultures only. As shown in Figure 2, cell death by erlotinib was associated with caspase- 8 activation in 3D-cultured cells. Caspase- 8 is an initiator caspase that is activated by receptormediated apoptosis, such as that induced by Fas, TRAIL, or TNF- $\alpha$. Therefore, we examined the expression of TRAIL and Fas after erlotinib treatment. Interestingly, treatment with erlotinib induced the expression of TRAIL in 3D cell cultures but not in $2 \mathrm{D}$ cell cultures (Figure $3 \mathrm{~A}$ and $\mathrm{B}$ ). Next, we evaluated the effect of TRAIL expression on erlotinib-induced cell death in $3 \mathrm{D}$ cultures. We infected cells with lentiviral TRAIL shRNA and then, treated the cells with erlotinib. Down-regulation of TRAIL completely inhibited erlotinibinduced apoptosis, as measured by annexin- $\mathrm{V}$ positivity, in 3D cultures (Figure 3C and D). Consistently, after TRAIL shRNA treatment, cleavage of caspase- 8 and caspase- 3 was inhibited in $3 \mathrm{D}$ cultures exposed to erlotinib (Figure $3 \mathrm{E}$ and $\mathrm{F}$ ). Together, these results demonstrate that erlotinib induces apoptosis in cells grown in $3 \mathrm{D}$ cultures in a TRAIL-dependent manner.

In $3 D$ cultures, autophagy precedes activation of TRAIL after exposure to erlotinib. As shown in Figure 3, TRAIL is a key factor in cell death induction by erlotinib in $3 \mathrm{D}$ cultures. Accordingly, we investigated the molecular mechanisms underlying TRAIL up-regulation by erlotinib in 3D cultures. Autophagy was recently reported to be responsible for TRAIL activation following EGFR inhibition (18). Thus, we 


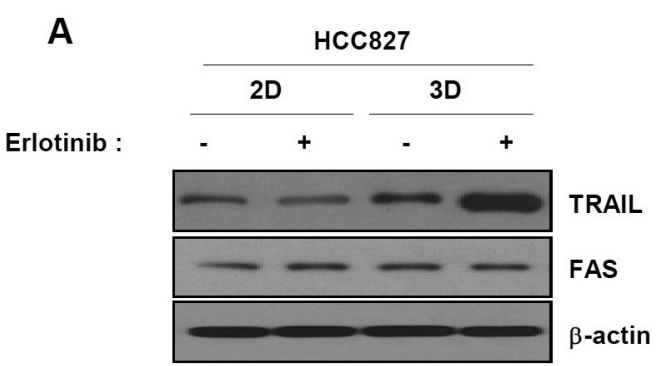

C

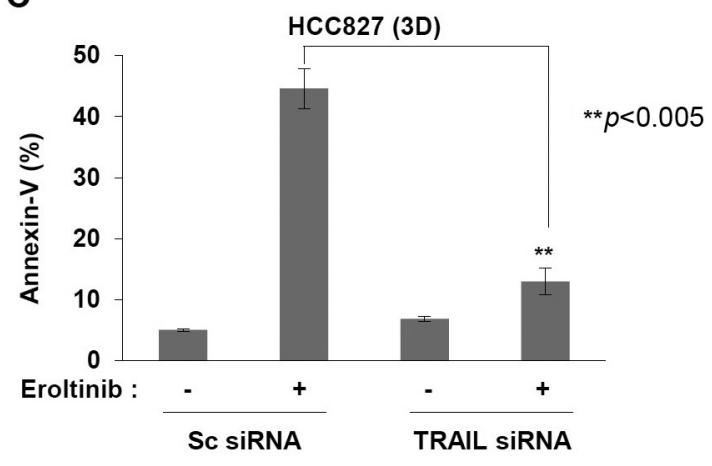

E

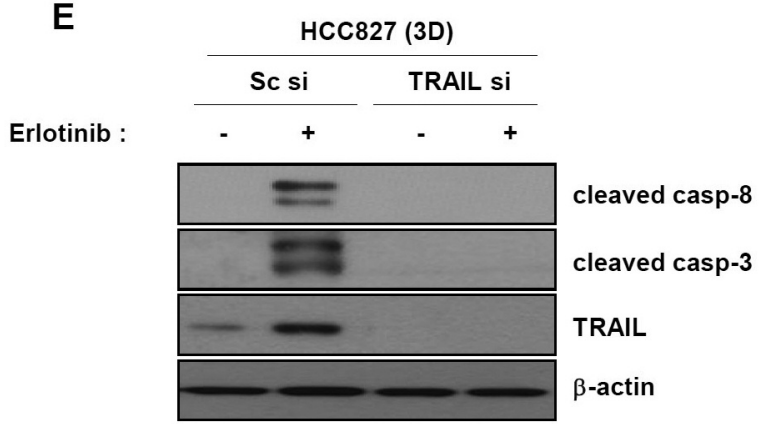

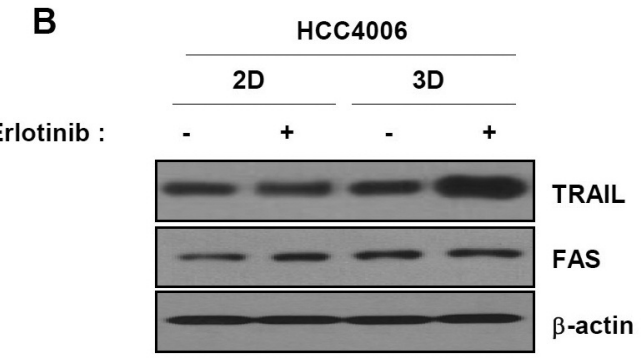

D

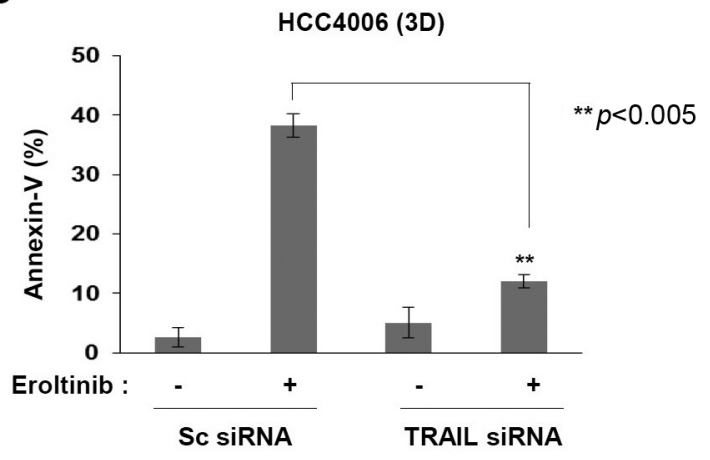

$\mathbf{F}$

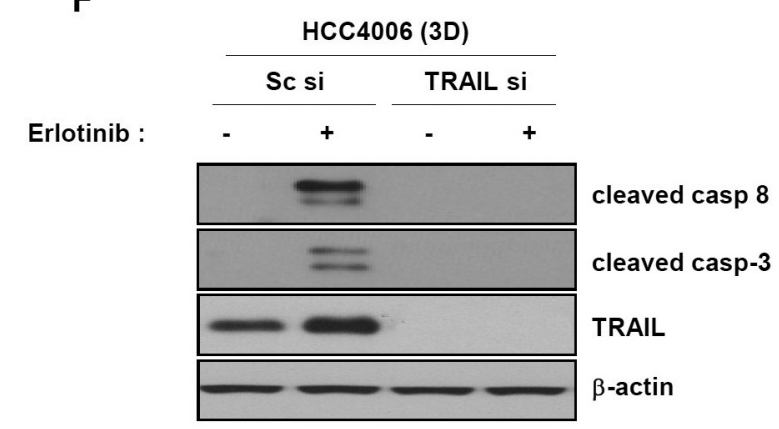

Figure 3. Erlotinib induces expression of TRAIL in 3D culture only. (A and B) HCC827 and HCC4006 cells grown under $2 D$ and $3 D$ culture conditions were treated with erlotinib for $24 \mathrm{~h}$. Cell lysates were subjected to immunoblot analysis with TRAIL or anti-Fas antibodies. $(C, D, E$ and F) HCC827 and HCC4006 cells grown under $3 D$ culture conditions were infected with scrambled or TRAIL shRNA lentivirus for 24 h, reseeded in poly-HEMA coated dish until spheroid formation at $72 \mathrm{~h}$, and then treated with $500 \mathrm{nM}$ erlotinib for $24 \mathrm{~h}$. (C and D) Cell death was measured by annexin-V staining using FACS. Error bars represent mean $\pm S . D$. of three separate experiments. ** $<0.01$. (E and $F)$ Expression of caspases and TRAIL was determined by immunoblot analysis using anti-cleaved caspase-8, anti-cleaved caspase-3, or anti-TRAIL. $\beta$-actin was used as a loading control.

examined the effect of autophagy on the response to erlotinib. Levels of the LC3II protein, which is an autophagy marker, were dramatically increased in response to erlotinib treatment in 3D but not 2D cultures (Figure 4A and B). Next, we examined the effect of autophagy on the expression of TRAIL following erlotinib treatment. We treated cells with 3-MA, an autophagy chemical inhibitor, before treatment with erlotinib. Up-regulation of TRAIL by erlotinib in 3D cultures was completely inhibited by 3-MA (Figure 4C and D). These data indicate that erlotinib induces TRAIL-mediated apoptotic cell death through activation of autophagy in $3 \mathrm{D}$ cultures.
JNK plays an important role in erlotinib-induced cell death in $3 D$ cultures. C-Jun N-terminal kinase (JNK) is associated with apoptosis induced by various death receptor ligands, including TRAIL and TNF- $\alpha(22,23)$. To determine whether phosphorylation of JNK influenced cell death by erlotinib in 3D cultures, we evaluated the level of phosphorylated JNK in erlotinib-treated cultures. Phosphorylation of JNK occurred in erlotinib-treated 3D but not 2D cultures (Figure $5 \mathrm{~A}$ and $\mathrm{B})$. We further analyzed the effect of JNK phosphorylation on erlotinib-induced cell death using a JNK chemical inhibitor, SP600125. Treatment of 3D cultures with 
A

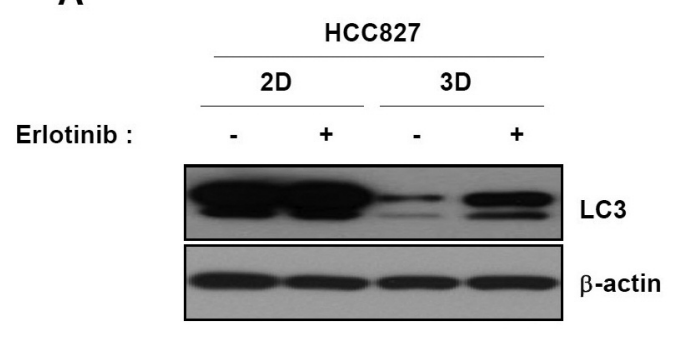

C

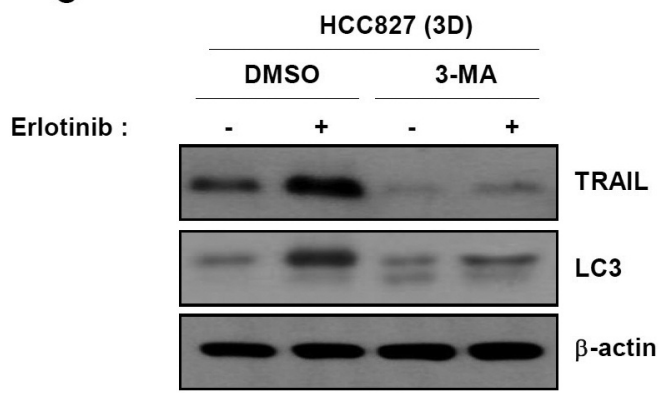

B

Erlotinib :

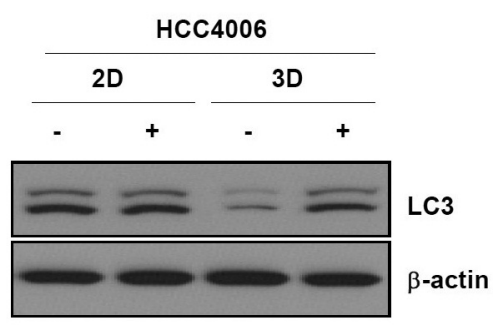

D

Erlotinib :

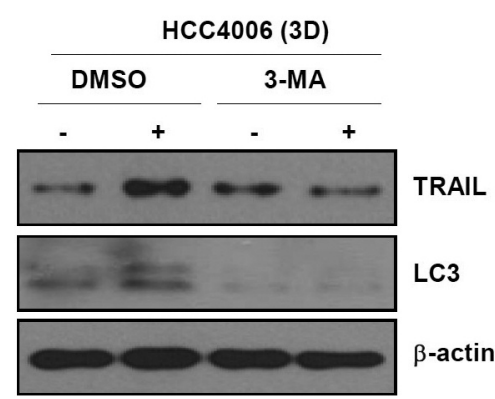

Figure 4. Up-regulation of TRAIL by erlotinib in $3 D$ cultures is mediated by autophagy. (A and B) Cells were treated with erlotinib and subjected to immunoblot analysis using anti-LC3 as a marker of autophagy. (C and D) 3D spheroid cells were pre-treated with 3-MA, an autophagy inhibitor, for $1 \mathrm{~h}$ and then treated with erlotinib for another $24 \mathrm{~h}$. Cell lysates were subjected to immunoblot analysis with anti-TRAIL or LC3 antibodies. Results are representative of three independent experiments.

SP600125 blocked erlotinib-induced cell death (Figure 5C and D). Consistently, activation of caspase-3, but not activation of TRAIL, by erlotinib in 3D cultures was abrogated in SP600125-treated cells (Figure 5E and F). These results indicate that erlotinib-induced apoptotic cell death in 3D cultures likely involves sequential activation of TRAIL-JNK pathways.

\section{Discussion}

Prior studies have focused on the molecular mechanisms of erlotinib in NSCLC 2D cell culture systems $(24,25)$. However, the mechanism by which erlotinib induces cell death in NSCLC 3D culture systems has not been examined. Such studies might be clinically relevant because it is recognized that $3 \mathrm{D}$ cultures better reflect the situation in vivo $(26,27)$. Therefore, an understanding of the response to erlotinib in 3D cultures could result in the development of new clinical anti-cancer strategies for NSCLC patients. In this study, we found that the molecular mechanisms of erlotinib-induced cell death in 2D and 3D cultures differed.

It has been reported that tumor cells grown as 3D spheroids are more resistant to chemotherapeutic agents or targeted agents than 2D cells, and new combinational anticancer strategies for $3 \mathrm{D}$ spheroid tumor cells have been developed by studying the underlying molecular mechanisms (28-30). Based on these reports, we investigated the sensitivity of cells grown in $2 \mathrm{D}$ and $3 \mathrm{D}$ cultures to erlotinib. In contrast with previous studies, we found that NSCLC cells grown in $2 \mathrm{D}$ and $3 \mathrm{D}$ cultures showed no difference in sensitivity to erlotinib.

We then examined whether there was a difference in the cell death pathway activated by erlotinib in $2 \mathrm{D}$ and $3 \mathrm{D}$ cultures. Interestingly, the active form of caspase- 8 was found only in 3D cultures after exposure to erlotinib. Moreover, blockade of caspase- 8 resulted in resistance to erlotinib-induced cell death in 3D cultures only, suggesting that cell death in response to erlotinib is induced by different caspase cascades in $2 \mathrm{D}$ culture. In addition to TRAIL, TNF$\alpha$ and Fas signaling pathways have been reported to initiate receptor-mediated apoptosis (31-33). We found that erlotinib elicited the expression of TRAIL, but not Fas, in 3D-cultured cells. Consistent with this observation, down-regulation of TRAIL completely abrogated erlotinib-induced caspase- 8 activation and subsequent cell death in 3D cultures, implying that erlotinib induces apoptotic cell death via TRAILcaspase- 8 activation.

We further found that up-regulation of TRAIL by erlotinib was strongly associated with the induction of autophagy in $3 \mathrm{D}$ cultures. Treatment of colon cancer cells with gefitinib 

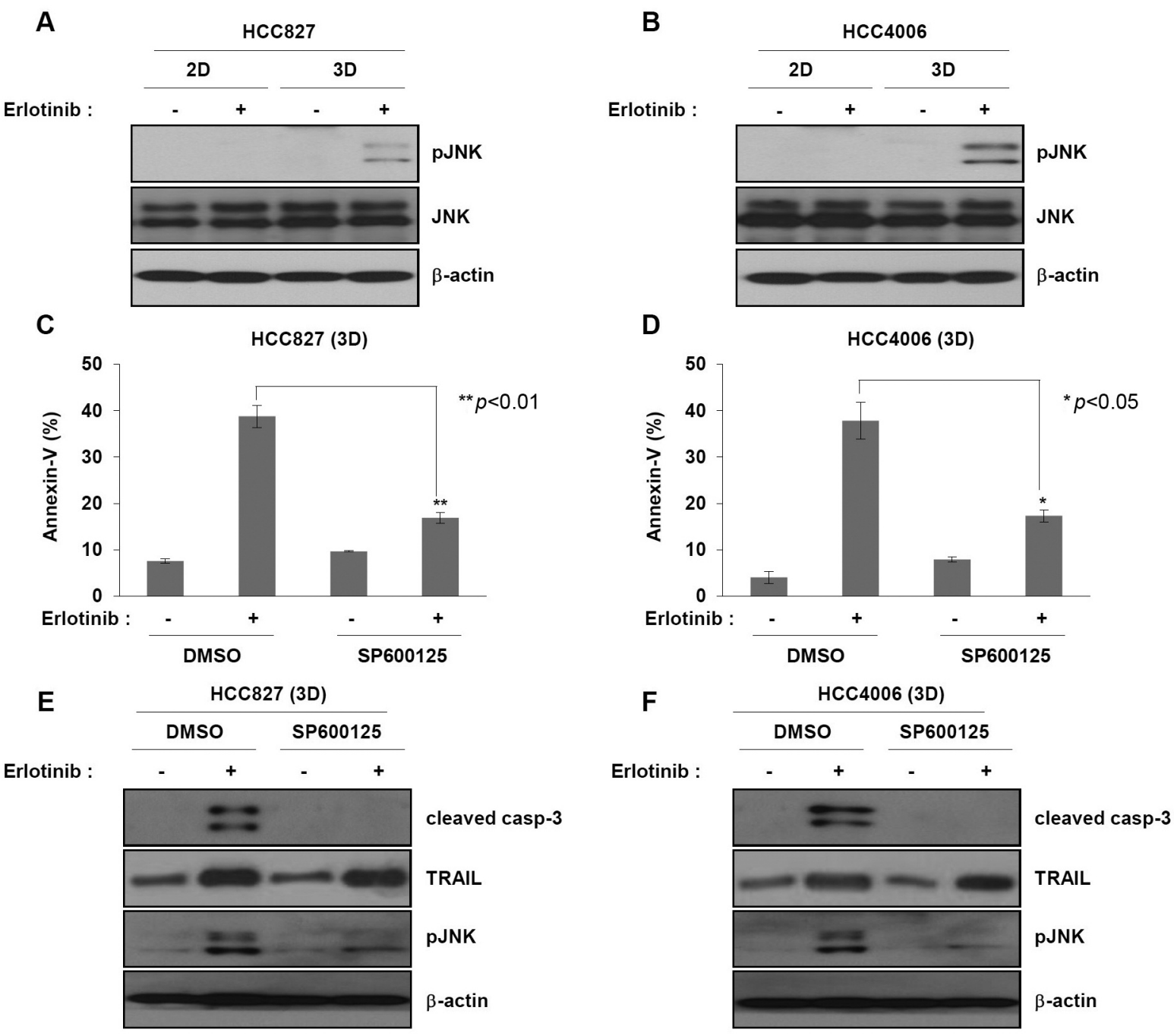

Figure 5. JNK plays an important role in erlotinib-induced cell death of non-small cell lung cancer cells grown in 3D culture. (A and B) HCC827 and HCC4006 cells grown in $2 \mathrm{D}$ or $3 \mathrm{D}$ were treated with $500 \mathrm{nM}$ erlotinib for $24 \mathrm{~h}$. They were subjected to immunoblot analysis to determine the levels of phosphorylated JNK using anti-phospho JNK antibody. (C, D, E and F) HCC827 and HCC4006 3D spheroid cells were pretreated with $10 \mu M$ SP600125, a JNK chemical inhibitor, for 1 h before treatment with $500 \mathrm{nM}$ erlotinib for $24 \mathrm{~h} .(\mathrm{C}$ and $D)$ Cell death was measured by annexin$V$ staining followed by FACS analysis. Graphs represent mean $\pm S . D$. of three separate experiments. $* * p<0.01$. (E and F) Activation of caspase-3 and TRAIL was determined by immunoblot analysis using anti-cleaved caspase-3 or anti-TRAIL antibodies. Results are representative of three independent experiments.

has been reported to induce autophagy and TRAIL expression (17). Another study reported that autophagy suppressed erlotinib-induced apoptotic cell death in NSCLC cells, implying that autophagy can inhibit apoptotic cell death (18). Interestingly, it was reported that autophagic flux in $3 \mathrm{D}$, but not $2 \mathrm{D}$, mesothelioma models better represented autophagy by the actual tumor; thus, the findings in $3 \mathrm{D}$ are likely to be more relevant to human disease (34). In our study, using 3D spheroids, erlotinib induced cell death by upregulating TRAIL via autophagy. These findings suggest that up-regulation of autophagy or TRAIL might enhance the effect of erlotinib.

Several studies have reported that JNK acts as the main effector of TRAIL-induced apoptotic cell death in various cancers (23). On the basis of these reports, we investigated the role of JNK in erlotinib-induced cell death in $3 \mathrm{D}$ cultures. We observed that phosphorylation of JNK was dramatically increased in erlotinib-treated 3D cultures but not $2 \mathrm{D}$ cultures, and that erlotinib-induced apoptotic cell death was significantly decreased in JNK inhibitor-treated 


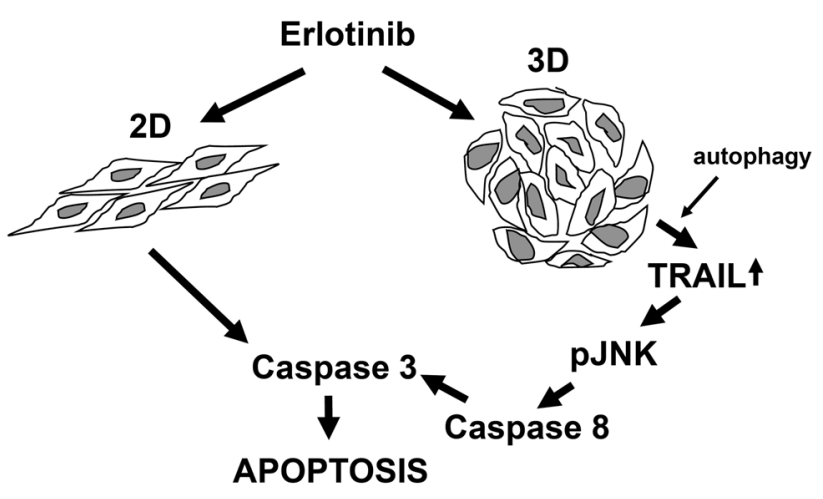

Figure 6. Suggested mechanism of Erlotinib-induced apoptosis in nonsmall cell lung cancer (NSCLC) 2D/3D culture. Although erlotinib treatment induces caspase3-dependent apoptotic cell death in both $2 D$ and $3 D$ cultures according to previous other studies using NSCLC $2 D$ cell culture systems, the activation caspase-8 was found only in $3 D$ cultures. Overall, our findings indicate that an autophagy-TRAIL-JNKcaspase pathway is involved in erlotinib-induced apoptosis of cells grown in $3 D$ culture.

3D culture cells, indicating that JNK plays a major role in erlotinib-induced apoptotic cell death in 3D cultures.

The 3D spheroid cell culture system has been proposed as an appropriate model of the in vivo tumor environment. In light of this, an understanding of the mechanism of action of erlotinib could facilitate development of novel anti-cancer therapies in NSCLC patients. Overall, our findings, as summarized in Figure 6, indicate that an autophagy-TRAILJNK pathway is involved in erlotinib-induced apoptosis of cells grown in 3D culture (Figure 6). Therefore, the effect of erlotinib in NSCLC patients can be enhanced by using TRAIL or other agonists, by activating JNK, or by stimulating autophagy. Further studies of erlotinib resistance in 3D cultured cells are needed to determine whether these approaches are helpful for forestalling or bypassing resistance to erlotinib.

\section{Conflicts of Interest}

The Authors declare no conflicts of interest in relation to this study.

\section{Authors' Contributions}

Conceptualization, Hyun-Kyung Lee and Dae Young Hur; Data curation, Min Hye Noh, Seung-Woo Hong, and Dae Young Hur; Formal analysis, Seung-Woo Hong, Seung-Mi Kim and Sung Hyun Kim; Funding acquisition, Dae Young Hur; Investigation, Min Hye Noh, Seung-Woo Hong and Seung-Mi Kim; Project administration, Hyun-Kyung Lee, V. Courtney Broaddus and Dae Young Hur; Writing - original draft, Seung-Woo Hong and Yeong Seok Kim; Writing - review \& editing, Hyun-Kyung Lee, V. Courtney Broaddus and Dae Young Hur.

\section{Acknowledgements}

This work was supported by the 2019 Inje University research grant. The Authors would like to thank Dario Barbone, $\mathrm{PhD}$, for his assistance with preliminary studies.

\section{References}

1 Bray F, Ferlay J, Soerjomataram I, Siegel RL, Torre LA and Jemal A: Global cancer statistics 2018: GLOBOCAN estimates of incidence and mortality worldwide for 36 cancers in 185 countries. CA Cancer J Clin 68(6): 394-424, 2018. PMID: 30207593. DOI: 10.3322/caac.21492

2 Tan WL, Jain A, Takano A, Newell EW, Iyer NG, Lim WT, Tan EH, Zhai W, Hillmer AM, Tam WL and Tan DSW: Novel therapeutic targets on the horizon for lung cancer. Lancet Oncol 17(8): e347-e362, 2016. PMID: 27511159. DOI: 10.1016/S14702045(16)30123-1

3 Soda M, Choi YL, Enomoto M, Takada S, Yamashita Y, Ishikawa S, Fujiwara S, Watanabe H, Kurashina K, Hatanaka H, Bando M, Ohno S, Ishikawa Y, Aburatani H, Niki T, Sohara Y, Sugiyama Y and Mano $\mathrm{H}$ : Identification of the transforming EML4-ALK fusion gene in non-small-cell lung cancer. Nature 448(7153): 561566, 2007. PMID: 17625570. DOI: 10.1038/nature05945

4 Ciardiello $\mathrm{F}$ and Tortora G: EGFR antagonists in cancer treatment. N Engl J Med 358(11): 1160-1174, 2008. PMID: 18337605. DOI: 10.1056/NEJMra0707704

5 Normanno N, Maiello MR, Chicchinelli N, Iannaccone A, Esposito C, De Cecio R, D'alessio A and De Luca A: Targeting the EGFR T790M mutation in non-small-cell lung cancer. Expert Opin Ther Targets 21(2): 159-165, 2017. PMID: 28002980. DOI: 10.1080/14728222.2017.1272582

6 Inal C, Yilmaz E, Piperdi B, Perez-Soler R and Cheng H: Emerging treatment for advanced lung cancer with EGFR mutation. Expert Opin Emerg Drugs 20(4): 597-612, 2015. PMID: 26153235. DOI: 10.1517/14728214.2015.1058778

7 Cohen MH, Johnson JR, Chen YF, Sridhara R and Pazdur R: FDA drug approval summary: erlotinib (Tarceva) tablets. Oncologist 10(7): 461-466, 2005. PMID: 16079312. DOI: 10.1634/theoncologist.10-7-461

8 Karachaliou N, Codony-Servat J, Teixidó C, Pilotto S, Drozdowskyj A, Codony-Servat C, Giménez-Capitán A, MolinaVila MA, Bertrán-Alamillo J, Gervais R, Massuti B, Morán T, Majem M, Felip E, Carcereny E, García-Campelo R, Viteri S, González-Cao M, Morales-Espinosa D, Verlicchi A, Crisetti E, Chaib I, Santarpia M, Luis Ramírez J, Bosch-Barrera J, Felipe Cardona A, de Marinis F, López-Vivanco G, Miguel Sánchez J, Vergnenegre A, Sánchez Hernández JJ, Sperduti I, Bria E and Rosell R: BIM and mTOR expression levels predict outcome to erlotinib in EGFR-mutant non-small-cell lung cancer. Sci Rep 5: 17499, 2015. PMID: 26639561. DOI: 10.1038/srep17499

9 Qian X, Li J, Ding J, Wang Z, Zhang W and Hu G: Erlotinib activates mitochondrial death pathways related to the production of reactive oxygen species in the human non-small cell lung cancer cell line A549. Clin Exp Pharmacol Physiol 36(5-6): 487-494, 2009. PMID: 19673930. DOI: 10.1111/j.1440-1681.2008.05091.x

10 Ryan SL, Baird AM, Vaz G, Urquhart AJ, Senge M, Richard DJ, O'Byrne KJ and Davies AM: Drug Discovery approaches utilizing three-dimensional cell culture. Assay Drug Dev Technol 14(1): 1928, 2016. PMID: 26866750. DOI: 10.1089/adt.2015.670 
11 Tanner K and Gottesman MM: Beyond 3D culture models of cancer. Sci Transl Med 7(283): 283ps9, 2015. PMID: 25877888. DOI: 10.1126/scitranslmed.3009367

12 Yang TM, Barbone D, Fennell DA and Broaddus VC: Bcl-2 family proteins contribute to apoptotic resistance in lung cancer multicellular spheroids. Am J Respir Cell Mol Biol 41(1): 1423, 2009. PMID: 19097992. DOI: 10.1165/rcmb.2008-0320OC

13 Wilson SM, Barbone D, Yang TM, Jablons DM, Bueno R, Sugarbaker DJ, Nishimura SL, Gordon GJ and Broaddus VC: mTOR mediates survival signals in malignant mesothelioma grown as tumor fragment spheroids. Am J Respir Cell Mol Biol 39(5): 576-583, 2008. PMID: 18511708. DOI: 10.1165/ rcmb.2007-0460OC

14 Barbone D, Yang TM, Morgan JR, Gaudino G and Broaddus VC: Mammalian target of rapamycin contributes to the acquired apoptotic resistance of human mesothelioma multicellular spheroids. J Biol Chem 283(19): 13021-13030, 2008. PMID: 18339627. DOI: $10.1074 /$ jbc.M709698200

15 Villanueva MT: Autophagy: Exploring the anticancer effects of autophagy inhibition. Nat Rev Cancer 15(9): 512-3, 2015. PMID: 26268833. DOI: 10.1038/nrc4003

16 Green DR and Levine B: To be or not to be? How selective autophagy and cell death govern cell fate. Cell 157(1): 65-75, 2014. PMID: 24679527. DOI: 10.1016/j.cell.2014.02.049

17 Chen L, Meng Y, Guo X, Sheng X, Tai G, Zhang F, Cheng H and Zhou Y: Gefitinib enhances human colon cancer cells to TRAIL-induced apoptosis of via autophagy- and JNK-mediated death receptors upregulation. Apoptosis 21(11): 1291-1301, 2016. PMID: 27629794. DOI: 10.1007/s10495-016-1287-5

18 Li YY, Lam SK, Mak JC, Zheng CY and Ho JC: Erlotinibinduced autophagy in epidermal growth factor receptor mutated non-small cell lung cancer. Lung Cancer 81(3): 354-361, 2013. PMID: 23769318. DOI: 10.1016/j.lungcan.2013.05.012

19 Ivascu A and Kubbies M: Rapid generation of single-tumor spheroids for high-throughput cell function and toxicity analysis. J Biomol Screen 11(8): 922-932, 2006. PMID: 16973921. DOI: $10.1177 / 1087057106292763$

20 Ekert JE, Johnson K, Strake B, Pardinas J, Jarantow S, Perkinson R and Colter DC: Three-dimensional lung tumor microenvironment modulates therapeutic compound responsiveness in vitro - implication for drug development. PLoS One 9(3): e92248, 2014. PMID: 24638075. DOI: 10.1371/journal.pone.0092248

21 Amann J, Kalyankrishna S, Massion PP, Ohm JE, Girard L, Shigematsu H, Peyton M, Juroske D, Huang Y, Stuart Salmon J, Kim YH, Pollack JR, Yanagisawa K, Gazdar A, Minna JD, Kurie JM and Carbone DP: Aberrant epidermal growth factor receptor signaling and enhanced sensitivity to EGFR inhibitors in lung cancer. Cancer Res 65(1): 226-235, 2005. PMID: 15665299

$22 \mathrm{Hu}$ WH, Johnson $\mathrm{H}$ and Shu HB: Tumor necrosis factor-related apoptosis-inducing ligand receptors signal NF-kappaB and JNK activation and apoptosis through distinct pathways. J Biol Chem 274(43): 30603-30610, 1999. PMID: 10521444. DOI: $10.1074 / \mathrm{jbc} .274 .43 .30603$

23 Sluss HK, Barrett T, Dérijard B and Davis RJ: Signal transduction by tumor necrosis factor mediated by JNK protein kinases. Mol Cell Biol 14(12): 8376-84, 1994. PMID: 7969172. DOI: $10.1128 / \mathrm{mcb} \cdot 14.12 .8376$
24 Costa DB, Halmos B, Kumar A, Schumer ST, Huberman MS, Boggon TJ, Tenen DG and Kobayashi S: BIM mediates EGFR tyrosine kinase inhibitor-induced apoptosis in lung cancers with oncogenic EGFR mutations. PLoS Med 4(10): 1669-1679; discussion 1680, 2007. PMID: 17973572. DOI: 10.1371/journal. pmed.0040315

25 Fung C, Chen X, Grandis JR and Duvvuri U: EGFR tyrosine kinase inhibition induces autophagy in cancer cells. Cancer Biol Ther 13(14): 1417-1424, 2012. PMID: 22954701. DOI: $10.4161 / \mathrm{cbt} .22002$

26 Yamada KM and Cukierman E: Modeling tissue morphogenesis and cancer in 3D. Cell 130(4): 601-610, 2007. PMID: 17719539. DOI: 10.1016/j.cell.2007.08.006

27 Marx V: Cell culture: a better brew. Nature 496(7444): 253-258, 2013. PMID: 23579682. DOI: $10.1038 / 496253 a$

28 Gomes LR, Vessoni AT and Menck CF: Three-dimensional microenvironment confers enhanced sensitivity to doxorubicin by reducing p53-dependent induction of autophagy. Oncogene 34(42): 5329-5340, 2015. PMID: 25619836. DOI: 10.1038/onc.2014.461

29 Torres-ayuso P, Daza-martín M, Martín-pérez J, Ávila-flores A and Mérida I: Diacylglycerol kinase $\alpha$ promotes 3D cancer cell growth and limits drug sensitivity through functional interaction with Src. Oncotarget 5(20): 9710-9726, 2020. DOI: 10.18632/ oncotarget.2344

30 Sakuma Y, Liu Q, Dubouzet JG, Abe H, Shinozaki K and Yamaguchi-Shinozaki K: DNA-binding specificity of the ERF/AP2 domain of Arabidopsis DREBs, transcription factors involved in dehydration- and cold-inducible gene expression. Biochem Biophys Res Commun 290(3): 998-1009, 2002. PMID: 11798174. DOI: $10.1006 /$ bbrc.2001.6299

31 Ashkenazi A and Dixit VM: Death receptors: signaling and modulation. Science 281(5381): 1305-1308, 1998. PMID: 9721089. DOI: 10.1126/science.281.5381.1305

32 Pan G, Ni J, Wei YF, Yu G, Gentz R and Dixit VM: An antagonist decoy receptor and a death domain-containing receptor for TRAIL. Science 277(5327): 815-818, 1997. PMID: 9242610. DOI: $10.1126 /$ science.277.5327.815

33 Kischkel FC, Hellbardt S, Behrmann I, Germer M, Pawlita M, Krammer PH and Peter ME: Cytotoxicity-dependent APO-1 (Fas/CD95)-associated proteins form a death-inducing signaling complex (DISC) with the receptor. EMBO J 14(22): 5579-5588, 1995. PMID: 8521815

34 Follo C, Barbone D, Richards WG, Bueno R and Broaddus VC: Autophagy initiation correlates with the autophagic flux in $3 \mathrm{D}$ models of mesothelioma and with patient outcome. Autophagy 12(7): 1180-1194, 2016. PMID: 27097020. DOI: 10.1080/ 15548627.2016 .1173799 